\title{
FIELD ASSESSMENT OF AUTODISSEMINATION OF PYRIPROXYFEN BY CONTAINER-INHABITING AEDES MOSQUITOES IN FLORIDA
}

\author{
BARRY W. ALTO*, SARA ORTIZ, KEENAN WIGGINS, CYNTHIA C. LORD, \\ AND NATHAN D. BURKETT-CADENA \\ University of Florida, IFAS, Florida Medical Entomology Laboratory, \\ 200 9th St. SE, Vero Beach, FL 32962
}

Subiect Editor: Rui-De Xue

\begin{abstract}
Domestic mosquito control for container-inhabiting Aedes vectors of Zika, chikungunya, yellow fever, and dengue viruses is challenging, and novel methods are needed. Autodissemination strategies are one such method. In this control method, females are attracted to stations treated with an insect growth regulator (IGR), become treated, and subsequently deposit the IGR in natural oviposition sites, preventing pupal emergence. We developed and tested treatment stations in semi-field conditions based on number of captured mosquitoes in the treatment stations. The modified treatment station attracted gravid females with oak leaf infusion and mosquito passage through exit chutes granted access to sucrose and topical contamination of IGR (pyriproxyfen) for $25 \%$ of released mosquitoes. Although a majority of released mosquitoes were uncaptured (75\%), sufficient amounts of pyriproxyfen contaminated female mosquitoes to result in $75 \%$ inhibition of adult emergence in larval bioassays. These stations were then used in a field experiment to test the efficacy using sentinel cups with mosquito larvae. Three treatments which included varied numbers of autodissemination stations (control, low density, and high density) were compared. Both low and high density of stations provided high inhibition of adult emergence from sentinel cups relative to controls. We did not observe differences in mosquito emergence inhibition whether sites contained low or high densities of stations (i.e., similar rates of mosquito inhibition). Two additional field trials showed that topically contaminated mosquitoes were traveling further than expected and causing mortality in sentinel cups at least as far as 80 meters from the autodissemination stations. The development and implementation of autodissemination of IGRs is an additional tool for use in integrated mosquito management.
\end{abstract}

Key Words: Domestic mosquito vectors, emerging mosquito-borne pathogens, vector control

\section{INTRODUCTION}

The global emergence of mosquitoborne pathogens such as Zika, dengue (DENV) and chikungunya (CHIKV) viruses in recent years have resulted in a large number of imported cases and local transmission of these three arboviruses in Florida, indicating increased risk for this region of the U.S. (Monaghan et al. 2016). These viruses are transmitted by invasive domestic mosquito species Aedes aegypti (L.) and Aedes albopictus (Skuse) which are widely dispersed throughout most of Florida (Lounibos et al. 2016). These two species have similar biological characteristics, including inhabiting containers during the immature stages and females visit multiple oviposition sites while laying eggs (skip oviposition) (Colton et al.
2003; Reiter 2007; Davis et al. 2015). Both species exhibit gonotrophic discordance whereby multiple blood feedings may occur during a single gonotrophic cycle, a behavior which enhances infection and transmission of arboviruses (Scott et al. 1993a, 1993b; Fernández and Forattini 2003). Small containers and hidden water-holding structures are notoriously difficult to find and treat using common control methods (Russell et al. 2002; Gonzalez et al. 1995; Montgomery \& Ritchie 2002; Barrera et al. 2008). However, mosquito control is the principal method to reduce human-mosquito-virus contact and reduce disease transmission, so developing improved methods to find and treat these containers is critical.

Autodissemination of insect growth regulators (IGRs) is a novel approach to deliv- 
ering insecticides to mosquito larval habitats through use of an autodissemination station (ADS) containing baits and insecticides such IGRs that disrupt development of mosquitoes during the immature stages. The use of IGRs that act on the late immature stages (pupae) takes advantage of other sources of mosquito mortality in nature that limit population sizes, including density-dependent mortality (Juliano 2007). For example, larval competition and nutrient limitation still occur and cause mortality prior to the mortality caused by the IGR at the pupal stage, resulting in higher overall immature mortality. Female mosquitoes are attracted to the stations when ready to lay eggs, become contaminated with the IGR and subsequently deposit it in other oviposition sites visited later (Kartzinel et al. 2016). Aedes aegypti and Ae. albopictus females exhibit skip-oviposition, increasing dissemination of the IGR (Colton et al. 2003; Reiter 2007; Davis et al. 2017, 2015). Pyriproxyfen has been used in several autodissemination studies because it is highly effective at low concentrations (Chism and Apperson 2003; Devine et al. 2009; Gaugler et al. 2012; Kartzinel et al. 2016; Unlu et al. 2017; Suman et al. 2018; Lwetoijera et al. 2019) and is not repellent to adult mosquitoes (Sihuincha et al. 2005). Previous studies have investigated different options for the medium used to carry the IGR, allow it to be picked up by females, and the level of active ingredient needed for acceptable levels of control (Gaugler et al. 2012; Wang et al. 2014; Kartzinel et al. 2016). However, many aspects of pyriproxyfen as a control method and autodissemination approaches warrant further research and development, in order to optimize station geometry, attractants, and IGR delivery to produce designs that treat large numbers of females and achieve high levels of control (Maoz et al. 2017). Important questions remain on the density of stations needed to achieve satisfactory control and distance over which autodissemination is effective (Suman et al. 2018). Here we describe studies aimed at improving and quantifying efficacy of a prototype autodissemination station for deployment of IGRs and assessment of the autodissemination stations in the field in Florida.

\section{MATERIALS AND METHODS}

\section{Autodissemination station optimization.}

The gravid Aedes (GAT) trap (Model 2797 BioQuip, Rancho Dominguez CA) was used as the overall structure of the station and was modified for each iteration of the autodissemination station development process. The capture chamber of the GAT trap (normally translucent) was painted black and a clear plastic funnel was placed at point of entry to reduce exit of females through the station entrance, a modification that was expected to increase the number of females that would exit through marking points. All stations utilized three exit chutes, consisting of vertically oriented tubes (Snap-Seal Disposable Plastic Sample Containers 02540-15, Fisher Scientific) that serve as the location for contaminating females with pyriproxyfen powder (Fig. 1).

A series of semi-field experiments, described below, were conducted to evaluate various station parameters and select more effective characteristics with the aim of designing an attractive station that "contaminated" a maximum number of females (i.e., individuals being tainted with pyriproxyfen). Variables examined included entry and exit modifications of the station, presence and absence of sugar source, types of lures, and level of contamination with pyriproxyfen. Gravid females (3 days post bloodmeal) of Ae. aegypti or Ae. albopictus (range 128-197) were released into a screened outdoor cage ( 2 x $2 \times 2 \mathrm{~m})$ with 2-3 prototype stations. Mosquitoes used in the studies were from laboratory lines established from field collections in Florida. Stations were checked daily for four consecutive days for trapped mosquitoes and to ensure the stations were functioning. In experiments 1-3, the number of females trapped was recorded daily, and the overall proportion of trapped females that were released was used to determine the relative effectiveness of modifications to the previous design. In experiment 4 , females were collected daily and used in larval bioassays 
A)

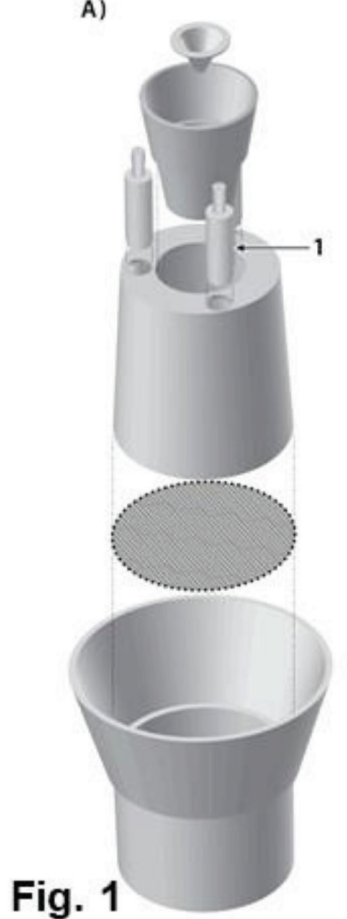

B)

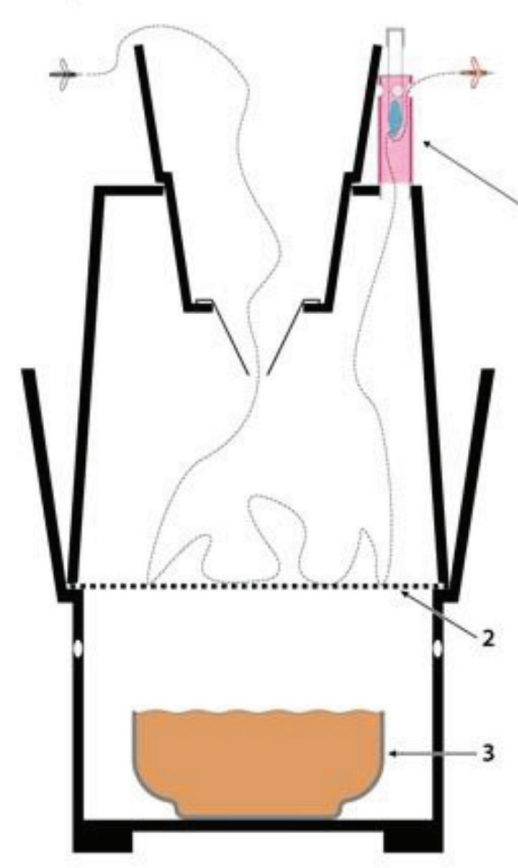

C)

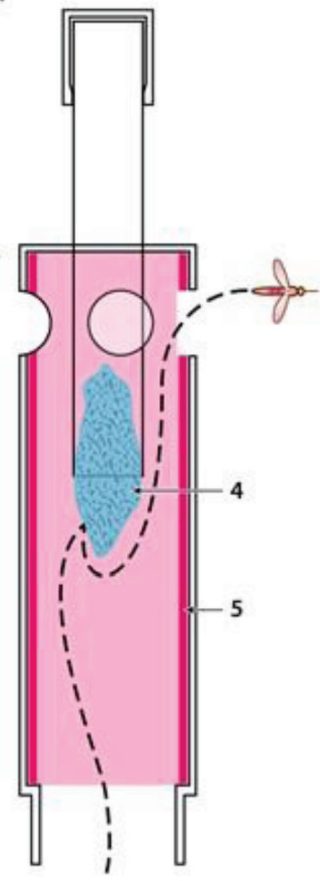

Figure 1. Autodissemination station components (A) with plastic exit chutes (1) containing pyriproxyfen. Mosquitoes enter the autodissemination station (B) and a screen (2) prevents them from access to oak leaf infusion attractant (3) and leave through the exit chute (C) which contains sucrose bait (4) and pyriproxyfen (5). Dashed lines denote the mosquito flight route.

to assess contamination with pyriproxyfen. For experiments 1-3, mesh bags were placed over exit chutes so that mosquitoes exiting through were captured for data recording (Fig. 1). Experiments were conducted over a period of four months (March-June 2016). We avoided contamination events by application of pyriproxyfen to exit chutes in a separate location compared to where seminatural and field trials were performed. All autodissemination station parts were handled with clean disposable gloves and pyriproxyfen treated items discarded after a single use.

Experiment 1 tested the effectiveness of stations using an oviposition lure (oak leaf water infusion) versus host-seeking lure (chemical lure described by Kartzinel et al. 2016). Ae. aegypti and Ae. albopictus exhibit gonotrophic discordance and so may feed more than once during a gonotrophic cycle, responding to host attractants while gravid. Three GAT traps (modified as above, with/ out exit nets) were deployed in two separate, consecutive trials. Each trial refers to a semi-field study. In trial 1 the oviposition lure consisted of $500 \mathrm{~mL}$ of well water mixed with $1000 \mathrm{~mL}$ oak leaf infusion (O'Meara et al. 1989). Oak leaf infusion, produced using published protocols (Alto et al. 2008), is routinely used to attract gravid Aedes mosquitoes for oviposition (Ponnusamy et al. 2010). Oak leaves are abundant and widespread in Florida container systems occupied by these Aedes mosquitoes (Reiskind et al. 2009). In trial 2 the host-seeking lure (chemical lure) consisted of a mixture of $1.0 \mathrm{~mL}$ hexanoic acid, $13.0 \mathrm{~mL}$ lactic acid, $26.0 \mathrm{~mL}$ tap water, and $2.3 \mathrm{~g}$ sodium polyacrylate (gel absorbent) and a separate container with $8 \mathrm{~g}$ ammonium bicarbonate (Kartzinel et al. 2016). Totals of 128 Ae. albopictus or Ae. aegypti females were released into the outdoor cage for each trial.

Experiment 2 examined the effects of infusion age and of adding a sugar source 
to the exit chute to draw females to the site of pyriproxyfen marking. Three GAT traps were deployed, each with three exit chutes (without pyriproxyfen) and associated "sugar wicks" in 2 trials. Each sugar wick consisted of a $5 \mathrm{~mL}$ plastic test tube with a cotton ball and $3 \mathrm{~mL}$ of $10 \%$ sucrose solution. The attractant was 1 liter of oak leaf infusion water (Trial 1, one week old and Trial 2, >2 weeks old) per station (O'Meara et al. 1989). Totals of 179 (Trial 2, >2 weeks old infusion) and 169 (Trial 1, 1-week-old infusion) Ae. albopictus females were released into the outdoor cages.

Experiment 3 tested the effect of adding pyriproxyfen to exit chutes (with sugar wicks) for trapping mosquitoes to assess if the presence of pyriproxyfen decreased attraction to the station. Aerosol cooking oil (PAM ${ }^{\circledR}$ Original) was applied ad libitum to the inner surface of each exit chute to improve adhesion of pyriproxyfen (Esteem ${ }^{\circledR}$ WP, Valent Biosciences) to mosquito cuticle. Two replicates were conducted. The first replicate employed three stations with 153 Ae. albopictus females. The second replicate employed two stations with 197 Ae. albopictus females. The attractant was 1 liter of oak leaf infusion water (one week old) per station (O'Meara et al. 1989). The goal of this experiment was simply to establish that mosquitoes would enter stations and pass through the exit chutes in the presence of pyriproxyfen, so no other treatments or controls were necessary. The subsequent experiment investigated application methods.

Experiment 4 tested the effect of two pyriproxyfen application methods for transfer of IGR to females, larval oviposition sites, and inhibition of adult emergence. Two GAT traps were deployed, each with 3 exit chutes, associated "sugar wicks" and pyriproxyfen. One station utilized exit chutes with the inner surface coated with aerosol cooking oil prior to pyriproxyfen (as in Experiment 3) while the other station utilized only pyriproxyfen dust ad libitum applied to the inner surface of the exit chutes. Untreated traps were not used as the goal was to compare treatment methods only. A total of 50 A . albopictus females were placed di- rectly into the station trap chamber and the entrance closed with cotton, so that females exiting the station would pass through the exit chutes and into collection bags. These females were freeze-killed then used in larval mortality bioassays using methods described in Kartzinel et al. (2016). Briefly, females were placed in WhirlPak bags containing $100 \mathrm{~mL}$ water, 10 2nd-instars of Ae. albopictus and larval food $(10 \mathrm{~mL}$ of food suspension consisting of equal parts yeast and lactalbu$\min )$. To avoid any potential for contamination, females from the same colony (never exposed to traps or the experimental cage) were freeze-killed and used as controls for the larval mortality bioassay. Bags were monitored for pupation, pupal death, and adult emergence.

For experiments 1-3, treatment effects (variables tested) on capture rates of mosquitoes in the autodissemination stations were analyzed using maximum likelihood categorical analyses of contingency tables (PROC CATMOD, SAS 2002) based on the number of mosquitoes recovered from the exit chutes. Some comparisons were within experiments while some were between experiments. Analysis of variance was used in experiment 4 to compare the two methods for pyriproxyfen application as measured by larval mortality bioassays, relative to controls (PROC GLM, SAS 2002). Tests of assumptions of normally distributed residuals and homogeneous variances were not significant. Post hoc tests used pairwise comparisons of treatment groups (Ryan-Einot-Gabriel-Welsch multiple stepdown procedure, SAS 2002).

\section{Field assessment of autodissemination station.}

Field experiments were performed at White City Cemetery, White City, Florida. The cemetery was chosen because it has been used in the past for collections of Ae. albopictus and Ae. aegypti and because it could be divided into areas with approximately similar environmental conditions (land cover and amount of shade). The experimental design designated three sections (approximately $60 \times 100 \mathrm{~m}$, with at least 20 $\mathrm{m}$ between sections) in the cemetery. The 
maximum distance $(100 \mathrm{~m})$ was consistent with the distance flown by most Ae. aegypti females, as determined by a comprehensive review of 21 mark-release-recapture studies of this vector (Harrington et al. 2005). The densities of ADS in each area were control (no stations), high density (18 stations) and low density (9 stations). The distance between treatment and control plots was approximately 80-150 meters. Stations were placed a $15 \times 50 \mathrm{~m}$ grid, with stations at each site for high and alternating in the long dimension for Low (Fig. 2). Ten sentinel larva cups (plastic cemetery vases, Kelco) were interspersed throughout the ADSs in the high and low treatments, and in the same relative locations in the control site. Sentinel cups were placed in the field containing $10 \mathrm{Ae}$. albopictus first instar larvae from a lab colony, larval food, and $250 \mathrm{ml}$ water. The field experiment was initiated 2 August 2016 with placement of the ADS and sentinel cups. Exit chutes were changed and pupae removed from sentinel cups 3 times/week. To minimize potential contamination, the control (no stations) sentinel cups were handled first when visiting the site. In treatment areas, one person collected sentinel cups while another changed exit chutes. This reduced the potential for contamination of sentinel cups by personnel during experimental monitoring. Stations were visually checked for any evidence of disturbance or potential contamination by dislodged treated exit chutes; none was observed during the experiment. Pupae from sentinel cups were held in the lab until eclosion and scored for successful adult emergence or mortality. The primary outcome variable was the proportion of pupae that died (as opposed to successfully emerging as adults) from each cup on each collection day. Adult mosquitoes in the field

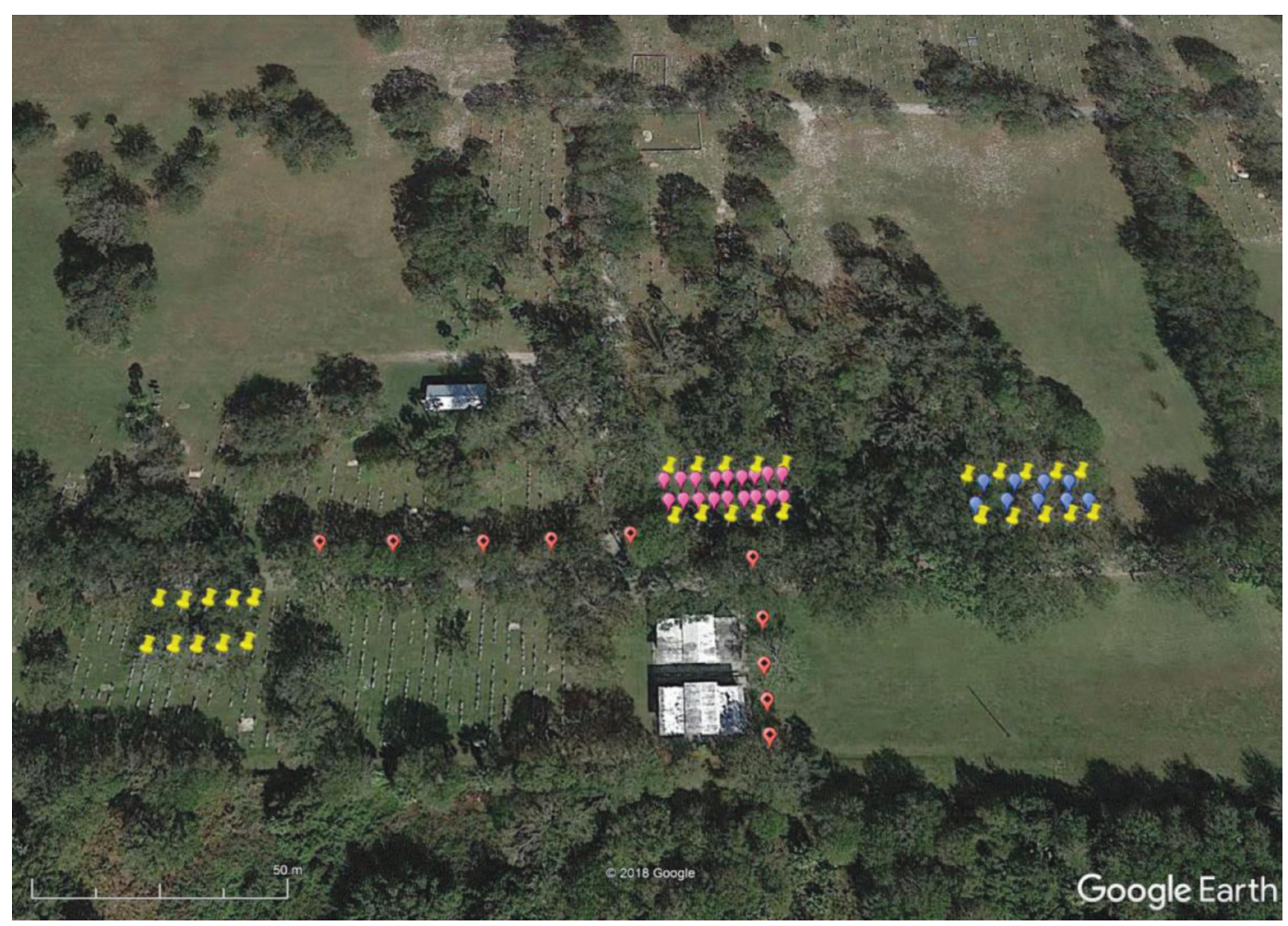

Figure 2. Aerial view of the cemetery with the experimental setup for treatment stations (high density, pink symbols; low density, blue symbols) and sentinel cups (yellow symbols) for the field experiment. Logistic constraints in the field situation required decreasing the long axis separation of the traps to approximately 6 meters. An array of sentinel cups (pink symbols with black dots) perpendicular to each other was used to evaluate the effect of distance of autodissemination station on mosquito mortality. 
laid eggs in the sentinel cups, enabling us to characterize mortality of natural mosquito populations in addition to those larvae originally placed in the cups. We performed two replicate trials of the field experiment in $\mathrm{Au}-$ gust and September 2016. An analysis of variance was used to test for a treatment effect of density of ADS, time (August and September runs of the field experiment) and density by time interaction on mosquito mortality using MatLab software.

We used a field experiment to investigate the maximum distance contaminated female mosquitoes are capable of transferring pyriproxyfen to container habitats and induce mosquito mortality. We set out an array of sentinel cups with sentinel Ae. albopictus larvae as described in the field assessment of autodissemination experiment. Ten sentinel cup "tripods" were split evenly between two perpendicular lines extending from one edge of the high area towards the control area and away from the experimental area. The tripods consisted of 3 sentinel cups, one closed by covering with a nitrile glove and 2 open. Immatures from the open cups for one tripod were combined when sampled. This allowed comparison of mortality when potentially pyriproxyfen-contaminated females did not (covered) or did (open) have access (Fig. 4). In short, cups with larvae were arranged along an array so contaminated adult mosquitoes could access them (open cups) or were prevented from access (covered cups). All tripods were placed in shade, limiting the regularity of placement, resulting in sentinel tripods at slightly different distances from the nearest ADS (Line 1: 9.83, 28.17, 43.36, 63.83 and 80.89m; Line 2: 9.88, 25.56, 36.66, 44.6 and $52.16 \mathrm{~m}$, Fig. 1). Tripods were in the field for 1 week, then pupal mortality assessed in the lab. Separate regression analyses were performed on proportion of pupae dead in covered (with nitrile glove) and open sentinel cups against distance from the nearest autodissemination station. Lines were combined for analysis. If regressions were significant, slopes were compared to assess differences between open and closed cups over distance. This was intended as a pre- liminary test of distance effects; however, a hurricane threat required dismantling the experiment and prevented further testing. Also, a separate two-tailed t-test compared mosquito mortality in open versus covered sentinel cups.

\section{RESULTS}

\section{Autodissemination station optimization.}

Experiment 1 tested the effectiveness of stations using an oviposition lure versus host-seeking lure. There were no significant effects of type of lure (host-seeking versus oviposition lure) on captures in the autodissemination stations $\left(\chi^{2}=2.24 ; \mathrm{df}=1 ; P\right.$ $=0.1343)$. The autodissemination stations captured between $9.4-15.6 \%$ of released mosquitoes (Table 1).

Experiment 2 examined the effects of infusion age and of adding a sugar source to the exit chute to attract mosquitoes to the site of pyriproxyfen marking. There were no significant effects of infusion age on capture rates in the autodissemination stations $\left(\chi^{2}\right.$ $=0.18 ; \mathrm{df}=1 ; P=0.6702)$. A total of 22.3$24.8 \%$ of released mosquitoes were captured in the exit chutes (Table 1). Although more mosquitoes were recovered in the exit chutes in experiment 2 than experiment 1 , this result was not significant $\left(\chi^{2}=0.68\right.$; $\mathrm{df}=$ $1 ; P=0.4095$, Table 1 ).

Experiment 3 tested the effect of adding pyriproxyfen to exit chutes for trapping mosquitoes to assess whether pyriproxyfen affects attraction to the station. There were no significant effects of addition of pyriproxyfen (pyriproxyfen or control) to exit chutes on captures $\left(\chi^{2}=0.05 ; \mathrm{df}=1 ; P=0.8241\right)$. A total of $21.3-28.8 \%$ of released mosquitoes were captured in the exit chutes in experiment 3 (Table 1).

Experiment 4 tested the effect of two pyriproxyfen application methods for transfer of IGR to females, larval oviposition sites and inducing larval mortality. The test for capture rates (females exiting the trap into collection bags) showed that significantly more females were recaptured when exit chutes contained oil plus IGR than IGR 
Table 1. Summary of experiments optimizing autodissemination station attractants and application methods in outdoor screen cage $(2 \times 2 \times 2 \mathrm{~m})$ using gravid Ae. albopictus and Ae. aegypti. Females either entered traps freely (lure, infusion age, sugar wick and IGR repellency) or were placed in traps and collected in exit chutes (application and mortality). Percentages are combined across multiple samples.

\begin{tabular}{|c|c|c|c|c|c|c|}
\hline Variable & Treatment & Species & $\mathrm{N}$ released & Metric & $\%$ & $\mathrm{P}$ \\
\hline \multirow[t]{2}{*}{ Lure } & Oviposition & Ae. albopictus & 128 & Recaptures & 15.6 & 0.1343 \\
\hline & Host & Ae. aegypti & 128 & & 9.4 & \\
\hline \multirow[t]{2}{*}{ Infusion age } & Old ( $>2$ week) & Ae. albopictus & 179 & Recaptures & 22.3 & 0.6702 \\
\hline & Fresh (1 week) & Ae. albopictus & 169 & & 24.8 & \\
\hline \multirow[t]{2}{*}{ Sugar wick } & Presence & Ae. albopictus & 169 & Recaptures & 24.8 & 0.4095 \\
\hline & Absence & Ae. albopictus & 128 & & 15.6 & \\
\hline \multirow[t]{2}{*}{ IGR repellency } & Presence & Ae. albopictus & 153 & Recaptures & 28.8 & 0.8241 \\
\hline & Absence & Ae. albopictus & 197 & & 21.3 & \\
\hline \multirow[t]{2}{*}{ Application method } & Oil + IGR & Ae. albopictus & 50 & Recaptures & 60 & 0.017 \\
\hline & Only IGR & Ae. albopictus & 50 & & 36 & \\
\hline \multirow[t]{2}{*}{ Control } & IGR & Ae. albopictus & 50 & Mortality & 24 & 0.0174 \\
\hline & No IGR & Ae. albopictus & 50 & & 70 & \\
\hline
\end{tabular}

only $\left(\chi^{2}=5.65 ; \mathrm{df}=1 ; P=0.0174\right.$, Table 1$)$. There were significant differences observed in the immature stage mortality in bioassays between pyriproxyfen treatment and control groups $\left(\mathrm{F}_{2,39}=5.2, P=0.009\right)$. A significantly lower percentage mosquitoes emerged to adulthood from the larval bioassays where females passed through exit chutes treated with aerosol cooking oil and pyriproxyfen (LS mean \pm SE, $24.0 \pm 5.8$ ) and pyriproxyfen dust (LS mean \pm SE, 27.7 \pm 6.1 ) than untreated controls (LS mean \pm SE, $70.0 \pm 13.1$ ). The addition of oil did not increase pyriproxyfen loading on mosquitoes because the two application methods did not significantly differ in immature mortality measured in bioassays (Table 1).

\section{Field assessment of autodissemination station.}

Two replicate trials of the field experiment were conducted during August and September 2016. Pupae were removed from sentinel cups 3 times/week. Figure 3 shows the proportion of mosquito pupae dead from the sentinel cups during the field trials. Results of ANOVA showed significant effects of density treatment (number of autodissemination stations), time (August or September), and density by time interaction (Table 2) on the proportion of pupae dying. The proportion of pupae dying was significantly different be- tween the density treatment groups, indicating an effect of the treatment stations. However, the two treatment densities were qualitatively similar, suggesting that the higher density of treatment stations did not improve control. In other words, both low and high numbers of autodissemination stations provide similar mosquito mortality in the sentinel cups. The interaction effect showed that these density treatment effects were observed in August but not in September (Fig. 3).

To determine the distance by which the autodissemination of IGR was effective, we set out an array of sentinel cups with Ae. albopictus larvae up to 80 meters from the treatment site containing autodissemination stations. Separate regressions were performed on proportion of pupae dead in covered and open sentinel cups against

Table 2. Analysis of variance testing for effects of density treatment of autodissemination stations, time (August and September runs of field experiment), and density by time interaction on pre-adult mortality of sentinel Ae. albopictus.

\begin{tabular}{lcrr}
\hline \hline Source & $d f$ & $F$-value & \multicolumn{1}{c}{$P$} \\
\hline Density treatment & 2 & 4.93 & 0.0109 \\
Time & 1 & 62.73 & $<0.0001$ \\
Density x Time & 2 & 3.18 & 0.0498 \\
Error & 52 & & \\
Total & 57 & & \\
\hline
\end{tabular}




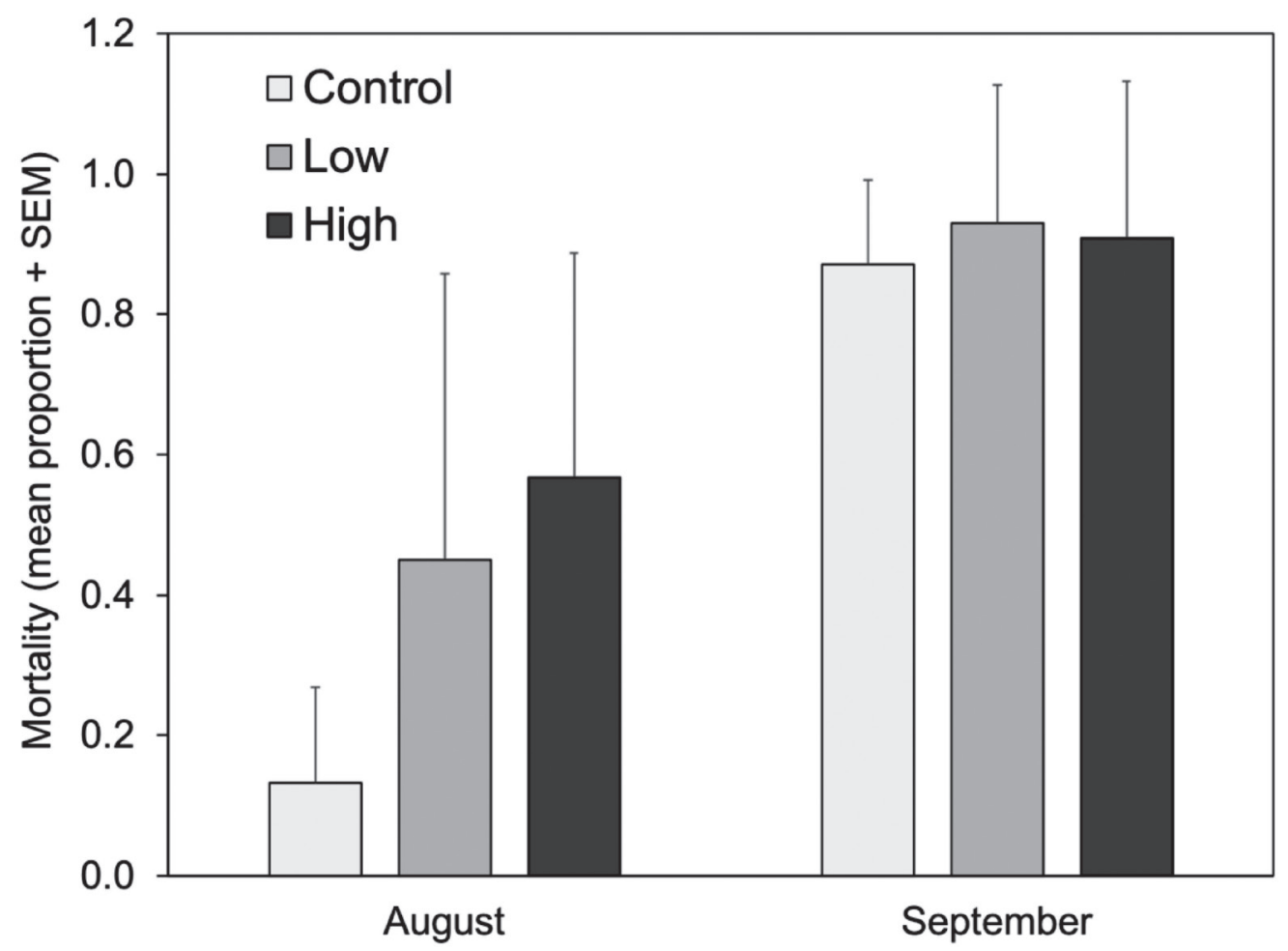

Figure 3. Effect of autodissemination station density on immature stage mortality of mosquitoes. Proportion dead pupae in sentinel cups from the field experiment in control (no stations) and treatment sites (low or high density) of autodissemination stations treated with pyriproxyfen. Two field trials were performed in August and September 2016.

distance. Neither regression was significant, indicating distance from the site with autodissemination stations did not affect pupal mortality in covered and open sentinel cups (Table 3). A separate test showed that mortality was higher in open sentinel cups than closed sentinel cups (two-tailed t-test, t18 = 2.2704, $P=0.0357$, Fig. 4). Increased mortality in open cups was observed up to $80 \mathrm{~m}$ from the treatment sites.

\section{DISCUSSION}

Here we report on the results of studies used to optimize an autodissemination station under semi-field conditions and two field trials of deployment to measure inhibition of adult emergence from sentinel larval cups. The best performing autodissemination station attracted gravid females with oak leaf infusion, although not significantly dif-

Table 3. Regression analyses on proportion of pupae dead in open and covered sentinel cups with Ae. albopictus against distance from a site with autodissemination stations.

\begin{tabular}{llcccc}
\hline \hline Sentinel cup & Source & Estimate & Standard Error & t-statistic & $P$ \\
Open & Intercept & 0.4639 & 0.2722 & 1.7041 & 0.1268 \\
& Distance & 0.0014 & 0.0061 & 0.2236 & 0.8287 \\
Error $d f$ & 8 & & & \\
Adjusted $r^{2}$ & -0.118 & & & 0.4164 \\
Covered & Intercept & 0.1883 & 0.2198 & 0.8570 & 0.8842 \\
& Distance & -0.0007 & 0.0049 & -0.1503 & \\
& Error $d f$ & 8 & & & \\
& Adjusted $r^{2}$ & -0.122 & & & \\
\hline
\end{tabular}




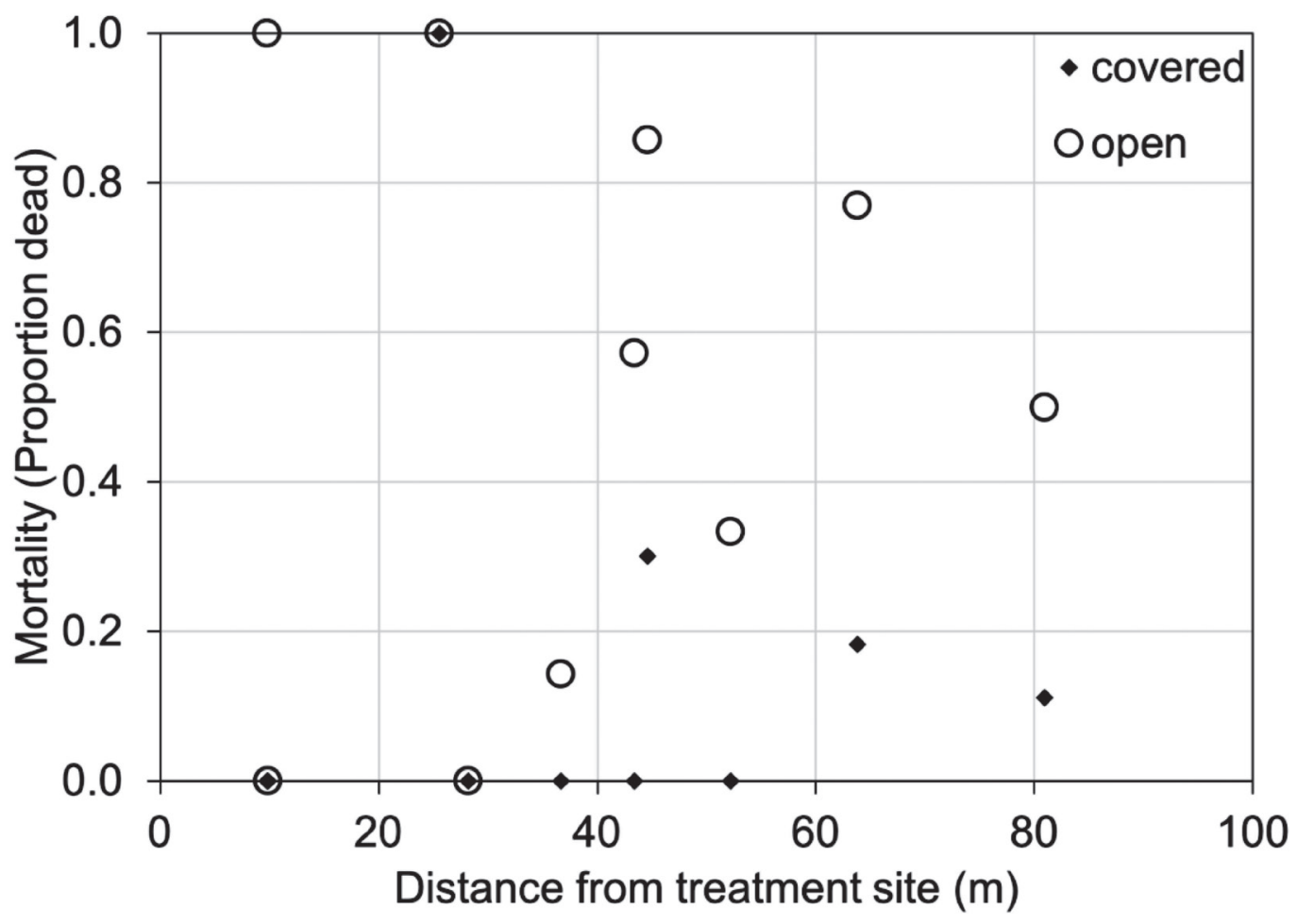

Figure 4. Relationship between distance and immature stage mosquito mortality in field assessment of autodissemination in Florida. Diamonds and circles represent covered (control) and open sentinel ovicups, respectively, used to monitor effect of pyriproxyfen on larval development. There was significantly higher mortality in open than covered sentinel ovicups (two-tailed t-test, t18 $=2.2704, \mathrm{p}=0.0357$ )

ferent from host-seeking lures. Mosquitoes enter the station through a funnel and are prevented access to the leaf infusion due to a screen barrier. Mosquitoes become topically contaminated with IGR when they leave the station through exit chutes that contain the IGR along with sucrose and oil. The narrow exit chute prevents mosquitoes from exiting by flight, and so mosquitoes are forced to walk through the exit chute which promotes tarsal contact with the IGR (Wang et al. 2014). Previous studies have shown that the efficacy of IGR rapidly declines over time under field conditions (Sullivan and Goh 2008; Kartzinel et al. 2016) and so there was a need to replace the IGR in stations. To address this issue, we included replaceable exit chutes for cost-effective and rapid renewal of IGRs in the autodissemination station without the need to move the station itself. This approach avoids the need for manipulation of pyriproxyfen and the station in the field. Rather, exit chutes can be prepared in ad- vance and in an environmentally controlled setting, avoiding issues of manipulating pyriproxyfen in wind and rain that could otherwise result in unintended effects on nontarget arthropods, such as pollinators.

Results from our semi-field trials showed that Ae. albopictus are attracted to the autodissemination stations. Our assessment of repellency of pyriproxyfen with Ae. albopictus confirmed previous findings for Ae. aegypti indicating lack of repellency (Sihuincha et al. 2005). Approximately $25 \%$ of released mosquitoes entered and passed through the exit chutes of the stations, ensuring topical contamination of pyriproxyfen. Low recaptures overall are due to mortality and escapes. The semi-field trials also demonstrated that sufficient amounts of pyriproxyfen topically contaminate female mosquitoes to result in approximately $75 \%$ adult emergence inhibition in bioassays. This level of emergence inhibition is similar to or higher than other observations in Ae. albopictus (Gaugler et al. 
2012; Caputo et al. 2012; Wang et al. 2014; Chandel et al. 2016; Kartzinel et al. 2016; Buckner et al. 2017; Unlu et al. 2017; Suman et al. 2018) and other mosquito species (Ae. aegypti, Devine et al. 2009; Buckner et al. 2017; Anopheles arabiensis, Lwetoijera et al. 2014).

We anticipated that the oil formulation would enhance topical contamination of mosquitoes and facilitate rapid release into the larval habitat upon contact (Chandel et al. 2016). However, the addition of oil did not appear to increase pyriproxyfen loading because the two application methods did not differ in immature mortality measured in bioassays (Table 1). We did not assess whether the retention time of pyriproxyfen differed between the two application methods, and so it is unclear whether the addition of oil may lengthen the time pyriproxyfen remains on adult mosquitoes. The addition of oil could minimize loss of pyriproxyfen on individuals attributable to grooming behaviors (Goldman et al. 1972; Golenda and Forgash 1986; Walker and Archer 1988; Jacquet et al. 2012).

The sentinel cups containing Ae. albopictus immature stages used small volumes of water $(250 \mathrm{~mL})$ so it is unclear how effective the approach of autodissemination of IGRs would be in larger volume containers. Topical contamination of adult mosquitoes with IGRs and subsequent transfer to large volume larval rearing sites is likely to result in substantial dilution of IGRs, and so control may be limited. However, Ae. albopictus seems to prefer small to medium sized containers (Hawley 1988; Carieri et al. 2003) and cemetery vases are common habitats (O'Meara et al. 1995). Achieving a lethal dose may not be an issue that compromises control of this invasive species.

A distinct advantage of the autodissemination control approach is that it exploits the oviposition behavior of gravid females in nature to locate and treat larval habitats with IGRs. Additionally, the ovicidal activity of pyriproxyfen limits the potential for topically contaminated mosquitoes to further contribute to mosquito populations (Suman et al. 2013, 2015), although induction depends on stage of ovarian development (Suman et al. 2015). For Ae. albopictus, oviposition in cryptic larval habitats may be favored over open containers, suggesting the former may make a substantial contribution to adult recruitment (Chandel et al. 2016). In many instances, conventional backpack and truck mounted application of larvicides are ineffective at penetrating cryptic larval habitats (Farajollahi et al. 2013; Achee et al. 2015), thus emphasizing the potential benefit of the autodissemination approach to target these mosquito habitats. We used open cups to assess efficacy of autodissemination of pyriproxyfen, and so additional studies are needed to evaluate immature stage mortality in cryptic larval habitats. A study on the efficacy of pyriproxyfen autodissemination assessed in residential areas showed similar or higher pupal mortality of Ae. albopictus in cryptic than open cup larval habitats (Chandel et al. 2016).

The efficacy of the autodissemination approach relies on several factors including the number of immature mosquitoes exposed to the IGR. This factor largely depends on oviposition behavior. Pheromone-like substances of larval and pupal origin have been shown in some instances to stimulate egg laying in containers inhabited by Aedes species (Ae. atropalpus, Kalpage and Brust 1973; Maire 1985; Ae.s togoi, Trimble and Wellington 1980; Ae. aegypti, Wong et al. 2011). So, the exploitation of mosquitoes for autodissemination benefits from mosquito behaviors that target container habitats suitable for development of the immature stages of mosquitoes (Wong et al. 2011). Further, skip oviposition, where females deposit eggs in multiple containers, allows for the possibility of multiple larval habitats to become contaminated with IGRs from a single topically contaminated female mosquito (Wang et al. 2014). However, in the current study, we did not specifically determine whether lethal doses of pyriproxyfen were transferred to multiple larval sites during skip oviposition.

Our work contributes to addressing several important aspects of autodissemination development, however several limitations in our study were apparent. The low percent- 
ages of recovered mosquitoes (less than $25 \%$ in most treatments) in outdoor screen cage assays, for example was perplexing. Despite exhaustive searching, the majority of remaining females could not be accounted for and were presumed to have been predated by ants or died and consumed by ants or other scavenging arthropods, however this could not be confirmed. Cages were checked daily during bioassays, which was done for convenience of regular sampling. Females were removed each time and net bags replaced. More frequent checking could have resulted in greater recapture rate. The reliance upon Ae. albopictus in nearly all bioassays to direct decisions regarding the development of the autodissemination station may not be warranted. While ecologically similar, differences in the biology of these mosquito species are recognized and could affect optimal station design. Full field trials would have benefitted from greater temporal spacing between major replicates to ensure no residual contamination of pyriproxyfen in the environment. In addition, greater distances between ADS and sentinel cups could permit investigating the maximum distance of pyriproxyfen dispersal by mosquitoes.

We observed differences in immature stage mortality between autodissemination station densities between the two field trials. In August, we observed enhanced inhibition of adult emergence in treatment sites with both low and high densities of autodissemination stations relative to the control site, suggesting that the higher density of treatment stations did not improve control. In September we observed much higher immature stage mortality at the field sites, including the control site. The efficacy of the autodissemination control strategy is correlated with the adult population size and so enhanced mortality in September may be attributable to greater numbers of adult mosquitoes. Surprisingly, we observed high immature stage mortality at control sites. We hypothesized that topically contaminated mosquitoes were moving further than anticipated and delivering pyriproxyfen to sentinel cups in the control site. To test this hypothesis, we performed an additional field study measuring immature stage mortality in sentinel cups along an array at increasing distances an autodissemination site. Distance from the site with autodissemination stations did not affect pupal mortality in covered and open sentinel cups with higher mortality in open sentinel cups than closed sentinel cups. Inhibition of adult emergence was observed at the furthest site $(80 \mathrm{~m})$, thus providing support for our hypothesis. A recent study showed that Ae. albopictus transferred pyriproxyfen from stations to contaminate containers and induced high immature stage mortality over $200 \mathrm{~m}$ (Suman et al 2018). Taken together, these results suggest that the autodissemination approach may extend the dissemination of IGR to larval habitats far from stations and substantially limit adult population sizes, especially in sites inaccessible to mosquito control personnel.

\section{ACKNOWLEDGEMENTS}

Jordan Vann assisted in the field experiment and James Newman assisted in preparation of figures. We thank the White City Cemetery board of directors and office manager Andrea Lawrence for allowing the field experiment at the cemetery. This study was supported by the Florida Department of Agriculture and Consumer Services contract number 022398.

\section{REFERENCES CITED}

Achee NL, Gould F, Perkins TA, Reiner Jr RC, Morrison AC, et al. 2015. A critical assessment of vector control for dengue prevention. PLoS Negl. Trop. Dis. 9(5): e0003655. doi 10.1371/journal.pntd.0003655.

Alto BW, Reiskind MH, Lounibos, LP. 2008. Size alters susceptibility of vectors to dengue virus infection and dissemination. Am. J. Trop. Med. Hyg, 79(5), 688695.

Barrera R, Amador M, Diaz A, Smith J, Munoz-Jordan JL, et al. 2008. Unusual productivity of Aedes aegypti in septic tanks and its implications for dengue control. Med. Vet. Entomol. 22:62-69.

Caputo B, lenco A, Cianci D, Pombi M, Petrarca V, Baseggio A, Devine JG, della Torre A. 2012. The "Auto-dissemination" approach: A novel concept to fight Aedes albopictus in urban areas. PLoS Negl. Trop. Dis. 6(8):e1793. doi:10.1371/journal.pntd.0001793.

Carrieri M, Bacchi M, Bellini R, Maini S. 2003. On the competition occurring between Aedes albopictus and Culex pipiens (Diptera: Culicidae) in Italy. Environ. Entomol. 32: 1313-1321. 
Chandel K, Suman DS, Wang Y, Unlu I, Williges E, Williams GM, R. Gaugler R. 2016. Targeting a hidden enemy: Pyriproxyfen autodissemination strategy for the control of the container mosquito Aedes albopictus in cryptic habitats. PLoS Negl. Trop. Dis. 10(12): e0005235. doi:10.1371/journal. pntd.0005235.

Chism BD, Apperson CS. 2003. Horizontal transfer of the insect growth regulator pyriproxyfen to larval microcosms by gravid Aedes albopictus and Ochlerotatus triseriatus mosquitoes in the laboratory. Med. Vet. Entomol. 17:211-220.

Colton YM, Chadee DD, Severson DW. 2003. Natural skip oviposition of the mosquito Aedes aegypti indicated by codominant genetic markers. Med. Vet. Entomol. 17: 195-204.

Davis TJ, Kaufman PE, Tatem AJ, Hogsette JA, Kline, DL. 2017. Development and evaluation of an attractive self-marking ovitrap to measure dispersal and determine skip oviposition in Aedes albopictus (Diptera: Culicidae) field populations. J. Med. Entomol. 53: 31-38.

Davis TJ, Kaufman PE, Hogsette JA, Kline DL. 2015. The effects of larval habitat quality on Aedes albopictus skip oviposition. J. AM. Mosq. Control Assoc. 31: 321-328.

Devine GJ, Perea EZ, Killeen GF, Stancil JD, Clark SJ, Morrison AC. 2009. Using adult mosquitoes to transfer insecticides to Aedes aegypti larval habitats. Proc. Natl. Acad. Sci. USA. 106:11530-11534.

Farajollahi A, Williams GM, Condon GC, Kesavaraju B, Unlu I, et al. 2013. Assessment of a direct application of two Bacillus thuringiensis israelensis formulations for immediate and residual control of Aedes albopictus. J. Am. Mosq. Control Assoc. 29:385388.

Fernández Z, Forattini OP. 2003. Survival of Aedes albopictus in Brazil: physiological age and reproductive history. Revista de Saúde Pública 37: 285-291.

Gaugler R, Suman D, Wang Y. 2012. An autodissemination station for the transfer of an insect growth regulator to mosquito oviposition sites. Med. Vet. Entomol. 26:37-45.

Goldman LJ, Callahan PS, Carlysle TC. 1972. Tibial combs and proboscis cleaning in mosquitoes. Ann. Entomol. Soc. Amer. 65:1299-1302.

Golenda CF, Forgash AJ. 1986. Grooming behavior in response to fenvalerate treatment in pyrethroidresistant house flies. Entomol. Exp. Appl. 40: 169175 .

Gonzalez R, Suarez MF. 1995. Sewers: The principal Aedes aegypti breeding sites in Cali, Colombia. Am. J. Trop. Med. Hyg. 53(Suppl 2):160.

Harrington LC, Scott TW, Lerdthusnee K, Coleman RC, Costero A, Clark GG, Jones JJ, Kitthawee S, Pattamaporn K, Sithiprasasna R, Edman JD. 2005. Dispersal of the dengue vector Aedes aegypti within and between rural communities. Am. J. Trop. Med. Hyg. 72:209-220.

Hawley WA. 1988. The biology of Aedes albopictus. J. Am. Mos. Control Assoc. 1: (Suppl.), 1-40.

Jacquet M, Lebon C, Lemperiere G, Boyer S. 2012. Behavioural functions of grooming in male Aedes albopictus (Diptera: Culicidae), the Asian tiger mosquito. Appl. Entomol. Zool. 47:359-363.

Juliano SA. 2007. Population dynamics. In: Floore TG, editor. Biorational Control of Mosquitoes. Supplement to the Journal of the American Mos- quito Control Association: American Mosquito Control Association Bulletin No. 7. pp. 265-275.

Kalpage DSP, Brust RA. 1973. Oviposition attractant produced by immature Aedes atropalpus. Environ. Entomol. 2:729-730.

Kartzinel MA, Alto BW, Deblasio MW II, BurkettCadena ND. 2016. Testing of visual and chemical attractants in correlation with the development and field evaluation of an autodissemination station for the suppression of Aedes aegypti and Aedes albopictus in Florida. J. Am. Mosq. Control Assoc. 32:194-202.

Lounibos LP, Bargielowski I, Carrasquilla MC, Nishimura N. 2016. Coexistence of Aedes aegypti and Aedes albopictus (Diptera: Culicidae) in Peninsular Florida two decades after competitive displacements. J. Med. Entomol. 53: 1385-1390.

Lwetoijera D, Kiware S, Okumu F, Devine GJ, Majambere S. 2014. Autodissemination of pyriproxyfen suppresses stable populations of Anopheles arabiensis under semi-controlled settings. Malaria J. 18:166.

Lwetoijera D, Harris C, Kiware S, Dpongus S, Devine GJ, McCall PJ, Majambere S. 2014. Effective autodissemination of pyriproxyfen to breeding sites by the exophilic malaria vector Anopheles arabiensis in semi-field settings in Tanzania. Malaria J. 13: 161.

Maire A. 1985. Effect of axenic larvae on the oviposition site selection by Aedes atropalpus. J. Am. Mosq. Control Assoc. 1:320-323.

Maoz D, Ward T, Müller P, Runge-Ranzinger S, Toledo J. et al. 2017. Community effectiveness of pyriproxyfen as a dengue vector control method: A systematic review. PLoS Negl. Trop. Dis. 11(7):e0005651.

Monaghan AJ, Morin CW, Steinhoff DF, Wilhelmi O, Hayden M, Quattrochi DA, Reiskind M, Lloyd AL, Smith K, Schmidt CA, Scalf PE, Ernst K. 2016. On the seasonal occurrence and abundance of the Zika virus vector mosquito Aedes aegypti in the contiguous United States. PLoS Curr. 16:8.

Montgomery BL, Ritchie SA. 2002. Roof gutters: A key container for Aedes aegypti and Ochlerotatus notoscriptus (Diptera: Culicidae) in Australia. Am. J. Trop. Med. Hyg. 67:244-246.

O'Meara GF, Vose FE, Carlson DB. 1989. Environmental factors influencing oviposition by Culex (Culex) (Diptera: Culicidae) in two types of traps. J. Med. Entomol. 26:528-534.

O'Meara GF, Evans LF, Gettman AD, Cuda JP. 1995. Spread of Aedes albopictus and decline of Ae. aegypti (Diptera: Culicidae) in Florida. J. Med. Entomol. 32:554-562.

Ponnusamy L, Xu N, Wesson DW, Ayyash LA, Schal C. Apperson CS. 2010. Oviposition response of the mosquitoes Aedes aegypti and Aedes albopictus to experimental plant infusions in laboratory bioassays. J. Chem. Ecol. 36:709-719.

Reiskind MH, Greene KL, Lounibos LP. 2009. Leaf species identity and combination affect performance and oviposition choice of two container mosquito species. Ecol. Entomol. 34:447-456.

Reiter P. 2007. Oviposition, dispersal, and survival in Aedes aegypti: Implications for the efficacy of control strategies. Vector-borne and Zoonotic Dis. 7: 261-273.

Russell BM, McBride JH, Mullner H, Kay BH. 2002. Epidemiological significance of subterranean A 
des aegypti breeding sites to dengue virus infection in Charters Towers 1993. J. Med. Entomol. 39:143145 .

Sihuincha M, E. Zamora-Perea W, Orellana-Rios J, Stancil V, Lopez-Sifuentes, et al. 2005. Potential use of pyriproxyfen for control of Aedes aegypti (Diptera: Culicidae) in Iquitos, Peru. J. Med. Entomol. 42:620-630.

Scott TW, Clark GG, Lorenz LH, Amerasinghe PH, Reiter P, Edman JD. 1993a. Detection of multiple blood feeding in Aedes aegypti (Diptera: Culicidae) during a single gonotrophic cycle using a histologic technique. J. Med. Entomol. 30:94-99

Scott TW, Chow E, Strickman D, Kittayapong P, Wirtz RA, Lorenz LH, Edman JD. 1993b. Blood-feeding patterns of Aedes aegypti (Diptera: Culicidae) collected in a rural Thai village. J. Med. Entomol. 30:922-927.

Sullivan JJ, Goh KS. 2008. Environmental fate and properties of pyriproxyfen. J. Pestic. Sci 33: 339350.

Suman DS, Wang Y, Faraji A, Williams GM, Williges E, Gaugler R. 2018. Seasonal field efficacy of pyriproxyfen autodissemination stations against container-inhabiting mosquito Aedes albopictus under different habitat conditions. Pest Manag. Sci. 74:885-895.

Suman DS, Wang Y, Gaugler R. 2015. The insect growth regulator pyriproxyfen terminates egg dia- pause in the Asian tiger mosquito, Aedes albopictus. PLoS ONE 10(6): e0130499.doi:10.1371/journal. pone.0130499.

Suman DS, Wang Y, Bilgrami AL, Gaugler R. 2013. Ovicidal activity of three insect growth regulators against Aedes and Culex mosquitoes. Acta Trop. 128:103-109.

Trimble RM, Wellington WG. 1980. Oviposition stimulant associated with fourth-instar larvae of $\mathrm{Ae}$ des togoi (Diptera: Culicidae). J. Med. Entomol. 17:509-514.

Unlu I, Suman DS, Wang Y, Klingler K, Faraji A, Gaugler A. 2017. Effectiveness of autodissemination stations containing pyriproxyfen in reducing immature Aedes albopictus populations. Parasites and Vectors 10:139.

Walker ED, Archer WE. 1987. Sequential organization of grooming behaviors of the mosquito, Aedes triseriatus. J. Insect Behav. 1:97-109.

Wang Y, Suman DS, Bertrand J, Dong L, Gaugler R. 2014. Dual-treatment autodissemination station with enhanced transfer of an insect growth regulator to mosquito oviposition sites. Pest Manag. Sci. 70:1299-1304.

Wong J, Stoddard ST, Astete H, Morrison AC, Scott TW. 2011. Oviposition site selection by the dengue vector Aedes aegypti and its implications for dengue control. PLoS Negl. Trop. Dis. 5(4): e1015. doi:10.1371/journal.pntd.0001015. 